\title{
INTUITIONISTIC FUZZY QUICK IDEALS IN $d$-ALGEBRAS
}

\author{
Sun Shin Ahn and Gyeong Ho Han
}

\begin{abstract}
Quick ideals and the fuzzification of quick ideals in $d$ algebras are considered, and some related properties are investigated. The intuitionistic fuzzification of quick ideals of a $d$-algebra is established, and related results are studied. The notion of equivalence relations on the family of all intuitionistic fuzzy quick ideals of a $d$-algebra is introduced, and then some properties are discussed.
\end{abstract}

\section{Introduction}

Y. Imai and K. Iséki introduced two classes of abstract algebras: $B C K$-algebras and $B C I$-algebras $([3,4])$. It is known that the class of $B C K$-algebras is a proper subclass of the class of $B C I$-algebras. J. Neggers and H. S. Kim ([9]) introduced the notion of $d$-algebras which is another useful generalization of $B C K$-algebras, and investigated several relations between $d$-algebras and $B C K$-algebras. In the same paper they also investigated other relations between $d$-algebras and oriented digraphs. J. Neggers, Y. B. Jun and H. S. Kim ([10]) discussed ideal theory in $d$-algebras, and introduced the notions of $d$-subalgebra, $d$-ideal, $d^{\#}$-ideal and $d^{*}$-ideal, and investigated some relations among them. Y. C. Lee and H. S. Kim ([7]) introduced the notion of $d$-transitive $d^{*}$ algebra which is another interesting generalization of $B C K$-algebras. Y. B. Jun, H. S. Kim and D. S. Yoo ([5]) established the notion of intuitionistic fuzzy $d$-algebra $X$ and obtained the fundamental results needed to develop a further theory of these objects.

In this paper, we introduce the notion of quick ideals and the fuzzification of quick ideals in $d$-algebras, and investigate some related properties in $d$-algebras. We also discuss the product of fuzzy quick ideals and

Received July 13, 2009. Accepted September 9, 2009.

2000 Mathematics Subject Classification. 06F35, 03G25.

Key words and phrases. (fuzzy) quick ideals, (fuzzy) $d$-subalgebra, (fuzzy) $d$ ideals. 
projections of fuzzy quick ideals in $d$-algebras. We establish the notion of intuitionistic fuzzy $B C K$-ideal $/ d$-ideal/quick ideal of a $d$-algebra and the notion of equivalence relations on the family of all intuitionistic fuzzy quick ideals of a $d$-algebra. We also discuss some related properties.

\section{Preliminaries}

A $d$-algebra $([9])$ is a non-empty set $X$ with a constant 0 and a binary operation " $*$ " satisfying axioms :

(I) $x * x=0$,

(II) $0 * x=0$,

(III) $x * y=0$ and $y * x=0$ imply $x=y$ for all $x, y \in X$.

A $B C K$-algebra is a $d$-algebra $(X ; *, 0)$ satisfying additional axioms:

(IV) $((x * y) *(x * z)) *(z * y)=0$,

(V) $(x *(x * y)) * y=0$, for all $x, y, z \in X$.

For brevity we also call $X$ a d-algebra. In $X$ we can define a binary relation " $\leq$ " by $x \leq y$ if and only if $x * y=0$. A mapping $\alpha: X \rightarrow Y$ of $d$-algebras is called a d-homomorphism if $\alpha(x * y)=\alpha(x) * \alpha(y)$ for all $x, y \in X$.

Definition 2.1. ([10]) Let $X$ be a $d$-algebra and let $\emptyset \neq I \subseteq X$. $I$ is called a $d$-subalgebra of $X$ if $x * y \in I$ whenever $x \in I$ and $y \in I$. $I$ is called a $B C K$-ideal of $X$ if it satisfies:

$\left(D_{0}\right) 0 \in I$,

$\left(D_{1}\right) x * y \in I$ and $y \in I$ imply $x \in I$.

$I$ is called a $d$-ideal of $X$ if it satisfies $\left(D_{1}\right)$ and

$\left(D_{2}\right) x \in I$ and $y \in X$ imply $x * y \in I$, i.e., $I * X \subseteq I$.

Definition 2.2. ([2]) An intuitionistic fuzzy set (IFS for short) $D$ in a $d$-algebra $X$ is an object having the form

$$
D=\left\{<x, \mu_{D}(x), \gamma_{D}(x)>\mid x \in X\right\}
$$

where the functions $\mu_{D}: X \rightarrow[0,1]$ and $\gamma_{D}: X \rightarrow[0,1]$ denote the degree of membership (namely $\mu_{D}(x)$ ) and the degree of nonmembership (namely $\gamma_{D}(x)$ ) of each element $x \in X$ to the set $D$, respectively, and where $0 \leq \mu_{D}(x)+\gamma_{D}(x) \leq 1$ for each $x \in X$.

For the sake of simplicity, we shall use the notation $D=<x, \mu_{D}, \gamma_{D}>$ instead of $D=\left\{<x, \mu_{D}(x), \gamma_{D}(x)>\mid x \in X\right\}$. Let $f$ be a mapping from a set $X$ to a set $Y$. If

$$
B=\left\{<y, \mu_{B}(y), \gamma_{B}(y)>\mid y \in Y\right\}
$$


is an IFS in $Y$, then the preimage of $B$ under $f$, denoted by $f^{-1}(B)$, is the IFS in $X$ defined by

$$
f^{-1}(B)=\left\{<x, f^{-1}\left(\mu_{B}\right)(x), f^{-1}\left(\gamma_{B}\right)(x)>\mid x \in X\right\},
$$

where $f^{-1}\left(\mu_{B}\right)(x)=\mu_{B}(f(x))$ and $f^{-1}\left(\gamma_{B}\right)(x)=\gamma_{B}(f(x))$ for all $x \in$ $X$.

\section{Quick ideals in $d$-algebras}

Definition 3.1. Let $X$ be a $d$-algebra and let $0 \in I \subseteq X$. $I$ is called a quick ideal of $X$ if for any $x, y \in X$ with $x * y \neq 0, x * y \in I$ implies $x, y \in I$.

The notion of a quick ideal is different from the notion of an ideal in $d$-algebras.

Example 3.2. Let $X:=\{0, a, b, c\}$ be a $d$-algebra ([6]) which is not a $B C K$-algebra with the following Cayley table:

\begin{tabular}{l|llll}
$*$ & 0 & $a$ & $b$ & $c$ \\
\hline 0 & 0 & 0 & 0 & 0 \\
$a$ & $a$ & 0 & 0 & $b$ \\
$b$ & $b$ & $c$ & 0 & $b$ \\
$c$ & $c$ & $c$ & $c$ & 0
\end{tabular}

Then $I:=\{0, a\}$ is a quick ideal of $X$ and a $B C K$-ideal of $X$.

Proposition 3.3. Let $X$ be a $d$-algebra and let $I$ be a quick ideal of $X$. Assume that $x * y=0, y \in I$ implies $x \in I$. Then $I$ is a $B C K$-ideal of $X$.

Proof. Assume that $x * y=0, y \in I$ implies $x \in I$. Then $I$ is a $B C K$-ideal of $X$ since $x * y \neq 0, x * y \in I$, also implies $x \in I, y \in I$.

Example 3.4. (1) Let $X:=\{0, a, b, c\}$ be a $d$-algebra ([10]) which is not a $B C K$-algebra with the Cayley table as follows:

\begin{tabular}{l|llll}
$*$ & 0 & $a$ & $b$ & $c$ \\
\hline 0 & 0 & 0 & 0 & 0 \\
$a$ & $a$ & 0 & 0 & $b$ \\
$b$ & $b$ & $b$ & 0 & 0 \\
$c$ & $c$ & $c$ & $c$ & 0
\end{tabular}

Then $A:=\{0, a\}$ is a quick ideal of $X$, a $B C K$-ideal of $X$, but not a $d$ ideal of $X$, since $a * c=b \notin A$. Also $A$ is a $d$-subalgebra of $X . B:=\{0, b\}$ is a $d$-subalgebra of $X$, but not a quick ideal of $X$, since $a * c=b \in B$, 
$a, c \notin B$. Also $B$ is not a $B C K$-ideal of $X$, since $a * b=0, b \in B$ and $a \notin B$.

(2) Let $X:=\{0, a, b, c\}$ be a $d$-algebra ([9]) which is not a $B C K$-algebra with the Cayley table as follows:

\begin{tabular}{c|cccc}
$*$ & 0 & $a$ & $b$ & $c$ \\
\hline 0 & 0 & 0 & 0 & 0 \\
$a$ & $a$ & 0 & 0 & $c$ \\
$b$ & $b$ & $b$ & 0 & 0 \\
$c$ & $c$ & 0 & $b$ & 0
\end{tabular}

Then $C:=\{0, a, c\}$ is a quick ideal of $X$, a $d$-subalgebra of $X$, but not a $B C K$-ideal of $X$ since $b * c=0, c \in C$ and $b \notin C$.

(3) Let $X:=\{0, a, b, c\}$ be a $d$-algebra ([9]) which is not a $B C K$-algebra with the Cayley table as follows:

\begin{tabular}{c|cccc}
$*$ & 0 & $a$ & $b$ & $c$ \\
\hline 0 & 0 & 0 & 0 & 0 \\
$a$ & $a$ & 0 & 0 & $b$ \\
$b$ & $b$ & $b$ & 0 & 0 \\
$c$ & $c$ & 0 & $b$ & 0
\end{tabular}

Then $D:=\{0, a, c\}$ is a quick ideal of $X$, but not a $d$-subalgebra of $X$ since $a * c=b \notin D$. $D$ is not a $B C K$-ideal of $X$, since $b * c=0, c \in D$ and $b \notin D$.

(4) Let $X:=\{0, a, b, c\}$ be a $d$-algebra which is not a $B C K$-algebra with the Cayley table as follows:

\begin{tabular}{l|llll}
$*$ & 0 & $a$ & $b$ & $c$ \\
\hline 0 & 0 & 0 & 0 & 0 \\
$a$ & $a$ & 0 & 0 & $b$ \\
$b$ & $b$ & $b$ & 0 & $b$ \\
$c$ & $c$ & $c$ & $c$ & 0
\end{tabular}

Then $E:=\{0, a, c\}$ is a $B C K$-ideal of $X$, not a quick ideal of $X$, since $c * b=c \in E$ and $b \notin E$. Also $E$ is not a $d$-subalgebra of $X$ since $a * c=b \notin E$.

\section{Fuzzy quick ideals in $d$-algebras}

Definition 4.1. ([6]) Let $\mu$ be a fuzzy set in a $d$-algebra $X$. Then $\mu$ is called a fuzzy d-subalgebra of $X$ if $\mu(x * y) \geq \min \{\mu(x), \mu(y)\}, \forall x, y \in X$. $\mu$ is called a fuzzy $B C K$-ideal of $X$ if

$\left(F_{0}\right) \mu(0) \geq \mu(x)$, 
$\left(F_{1}\right) \mu(x) \geq \min \{\mu(x * y), \mu(y)\}$, for all $x, y \in X$.

$\mu$ is called a fuzzy $d$-ideal of $X$ if it satisfies $\left(F_{1}\right)$ and

$\left(F_{2}\right) \mu(x * y) \geq \mu(x)$ for all $x, y \in X$.

Definition 4.2. Let $X$ be a $d$-algebra. A map $\mu: X \rightarrow[0,1]$ is called a fuzzy quick ideal of $X$ if it satisfies $\left(F_{0}\right)$ and

$\left(F_{3}\right)$ for any $x, y \in X$ with $x * y \neq 0, \min \{\mu(x), \mu(y)\} \geq \mu(x * y)$.

Theorem 4.3. Let $\mu$ be a fuzzy quick ideal of a $d$-algebra $X$. Assume $\mu$ is order reversing. Then $\mu$ is a fuzzy $B C K$-ideal of $X$.

Proof. Let $x, y \in X$. Since $\mu$ is order reversing, if $x * y=0$, then $\mu(x) \geq \mu(y)$. Hence $\mu(x) \geq \mu(y) \geq \min \{\mu(x * y)=\mu(0), \mu(y)\}$. If $x * y \neq 0$, then we have

$$
\mu(x) \geq \min \{\mu(x), \mu(y)\} \geq \mu(x * y) \geq \min \{\mu(x * y), \mu(y)\} .
$$

Therefore $\mu$ is a fuzzy $B C K$-ideal of $X$.

The converse of Theorem 4.3 need not be true.

Example 4.4. (1) Let $X:=\{0, a, b, c\}$ be a $d$-algebra as in Example 3.4-(2). Then $C:=\{0, a, c\}$ is a quick ideal of $X$, a $d$-subalgebra of $X$, but not a $B C K$-ideal of $X$. Define $\mu: X \rightarrow[0,1]$ be a fuzzy set defined by $\mu(0)=\mu(a)=\mu(c)=t_{1}$ and $\mu(b)=t_{2}$ for any $t_{1}, t_{2} \in[0,1]$ with $t_{1}>t_{2}$. Then $\mu$ is a fuzzy quick ideal of $X$ but not a fuzzy $B C K$-ideal of $X$ since $\mu(b)=t_{2} \leq \min \{\mu(b * c)=\mu(0), \mu(c)\}=t_{1} . \mu$ is not order reversing since $b * c=0$, i.e., $b \leq c$ implies $\mu(b)=t_{2} \leq \mu(c)=t_{1}$. Also $\mu$ is a fuzzy $d$-subalgebra of $X$.

(2) Let $X:=\{0, a, b, c\}$ be a $d$-algebra as in Example 3.4-(1). Then $F:=\{0, a, b\}$ is a $B C K$-ideal of $X$, not a quick ideal of $X$ since $a * c=$ $b, a \in F$ and $c \notin F$. Define $\rho: X \rightarrow[0,1]$ be a fuzzy set defined by $\rho(0)=\rho(a)=\rho(b)=t_{1}$ and $\rho(c)=t_{2}$. Then $\rho$ is a fuzzy $B C K$ ideal of $X$, order reversing, but not a fuzzy quick ideal of $X$, since $\min \{\rho(a), \rho(c)\}=\rho(c)=t_{2}<\rho(a * c)=\rho(b)=t_{1}$ for some $a * c=c \neq 0$.

Definition 4.5. Let $\mu$ be a fuzzy set in a set $X$. For $t \in[0,1]$, the set

$$
\mu_{t}:=\{x \in X \mid \mu(x) \geq t\}
$$

is called a level subset of $\mu$.

Theorem 4.6. Let $\mu$ be a fuzzy set of a $d$-algebra $X$. Then $\mu$ is a fuzzy quick ideal of $X$ if and only if for any $t \in[0,1]$ with $\mu_{t} \neq \emptyset, \mu_{t}$ is a quick ideal of $X$. 
Proof. For any $t \in[0,1]$ with $\mu_{t} \neq \emptyset$, let $x, y \in X$ with $x * y \neq 0$, $x * y \in \mu_{t}$. Then $\mu(x * y) \geq t$. Since $\mu$ is a fuzzy quick ideal of $X$, we have $\min \{\mu(x), \mu(y)\} \geq \mu(x * y) \geq t$. Hence $x, y \in \mu_{t}$. From $\left(F_{0}\right)$, we have $0 \in \mu_{t}$. Therefore $\mu_{t}$ is a quick ideal of $X$.

Conversely, assume that there exist $x, y \in X$ such that $x * y \neq 0$, $\mu(x * y)>\min \{\mu(x), \mu(y)\}$. Let $t_{1}:=\mu(x * y), t_{2}:=\mu(x), t_{3}:=\mu(y)$. Then $x * y \in \mu_{t_{1}}$, but at least one of $x$ or $y$ does not belong to $\mu_{t_{1}}$. Since $\mu_{t_{1}}$ is a quick ideal of $X$, we have $x, y \in \mu_{t_{1}}$, which is a contradiction.

Theorem 4.7. Let $I$ be a quick ideal of a $d$-algebra $X$ and let $\mu$ be a fuzzy set in $X$ defined by

$$
\mu(x)= \begin{cases}t_{0}, & \text { if } x \in I \\ t_{1}, & \text { if } x \notin I\end{cases}
$$

for all $x \in X$, where $t_{0}, t_{1} \in[0,1], t_{0}>t_{1}$. Then $\mu$ is a fuzzy quick ideal of $X$ and $\mu_{t_{0}}=I$.

Proof. Let $x, y \in X$ with $x * y \neq 0$. If any one of $x$ and $y$ does not belong to $I$, then $x * y \notin I$. Hence $\min \{\mu(x), \mu(y)\}=t_{1} \geq \mu(x * y)=t_{1}$. If $x, y \in I$, then $\mu(x)=\mu(y)=t_{0}$ and $\min \{\mu(x), \mu(y)\}=t_{0} \geq \mu(x * y)$. If $x, y \notin I$, then $x * y \notin I$. Hence $\min \{\mu(x), \mu(y)\}=t_{1}=\mu(x * y)$. Therefore $\mu$ is a quick ideal of $X$. It is clear that $\mu_{t_{0}}=I$.

Corollary 4.8. Any quick ideal of a d-algebra $X$ can be realized as a level subset of some fuzzy quick ideal of $X$.

The following theorem shows that the concept of a fuzzy quick ideal of $X$ is a generalization of a quick ideal. Using Corollary 4.8, the proof is straightforward.

Theorem 4.9. Let $I$ be a non-empty subset of a $d$-algebra of $X$ and let $\mu$ be a fuzzy set in $X$ such that $\mu$ is into $\{0,1\}$, so that $\mu$ is the characteristic function of $I$. Then $\mu$ is a fuzzy quick ideal of $X$ if and only if $I$ is a quick ideal of $X$.

Lemma 4.10. Let $\Lambda$ be a totally ordered set and let $\left\{I_{t} \mid t \in \Lambda\right\}$ be a family of quick ideals of $X$ such that for all $s, t \in \Lambda, s>t$ if and only if $I_{s} \subset I_{t}$. Then $\cap_{t \in \Lambda} I_{t}$ and $\cup_{t \in \Lambda} I_{t}$ are quick ideals of $X$.

Let $\Lambda$ be a non-empty subset of $[0,1]$.

Theorem 4.11. Let $\left\{I_{t}: t \in \Lambda\right\}$ be a collection of quick ideals of $X$ such that $X=\cup_{t \in \Lambda} I_{t}$ for all $s, t \in \Lambda, s>t$ if and only if $I_{s} \subset I_{t}$. Then a map $\mu$ defined by $\mu(x):=\sup \left\{t \mid x \in I_{t}\right\}$ for all $x \in X$ is a fuzzy quick ideal of $X$. 
Proof. Following Theorem 4.6, it is sufficient to show that $\mu_{s}$ is a quick ideal of $X$ for every $s \in[0,1]$. To do this, we divide into the following two cases:

(i) $s=\sup \{t \in \Lambda \mid t<s\}$ and

(ii) $s \neq \sup \{t \in \Lambda \mid t<s\}$.

Case (i) implies that $x \in \mu_{s} \Leftrightarrow x \in I_{t}$ for all $t<s \Leftrightarrow x \in \cap_{t<s} I_{t}$, so that $\mu_{s}=\cap_{t<s} I_{t}$, which is a quick ideal of $X$ by Lemma 4.10. For the case (ii), we claim that $\mu_{s}=\cup_{t \geq s} I_{t}$. If $x \in \cup_{t \geq s} I_{t}$, then $x \in I_{t}$ for some $t \geq s$. It follows that $\mu(x) \geq t \geq s$, so that $x \in \mu_{s}$. This proves that $\cup_{t \geq s} I_{t} \subseteq \mu_{s}$.

Now assume that $x \notin \cup_{t \geq s} I_{t}$. Then $x \notin I_{t}$ for all $t \geq s$. Since $s \neq \sup \{t \in \Lambda \mid t<s\}$, there exists $\epsilon>0$ such that $(s-\epsilon, s) \cap \Lambda=\emptyset$. Hence $x \notin I_{t}$ for all $t>s-\epsilon$, which means that if $x \in I_{t}$ then $t \leq s-\epsilon$. Thus $\mu(x) \leq s-\epsilon<s$, and so $x \notin \mu_{s}$. Therefore $\mu_{s} \subseteq \cup_{t \geq s} I_{t}$. Using Lemma 4.10, $\mu_{s}=\cup_{t \geq s} I_{t}$ is a quick ideal of $X$. Consequently, we conclude that $\mu$ is a quick ideal of $X$.

Definition 4.12. Let $\mu$ and $\nu$ be fuzzy quick ideals of $X_{1}$ and $X_{2}$, respectively. The product $\mu \times \nu$ of $\mu$ and $\nu$ is a fuzzy set of $X_{1} \times X_{2}$ which is defined by

$$
(\mu \times \nu):=\min \{\mu(x), \nu(y)\}, \forall(x, y) \in X_{1} \times X_{2} .
$$

Theorem 4.13. If $\mu_{i}$ are fuzzy quick ideals of $X_{i}(i=1,2)$, then $\mu_{1} \times \mu_{2}$ is a fuzzy quick ideal of $X_{1} \times X_{2}$.

Proof. For any $(x, y) \in X_{1} \times X_{2}$, we have $\left(\mu_{1} \times \mu_{2}\right)(0,0)=\mu\left\{\mu_{1}(0), \mu_{2}(0)\right\} \geq$ $\min \left\{\mu_{1}(x), \mu_{2}(y)\right\}=\left(\mu_{1} \times \mu_{2}\right)(x, y)$. The condition $\left(F_{0}\right)$ is true. Let $\left(x_{1}, x_{2}\right),\left(y_{1}, y_{2}\right) \in X_{1} \times X_{2}$ with $\left(x_{1}, x_{2}\right) *\left(y_{1}, y_{2}\right) \neq(0,0)$. Then

$$
\begin{aligned}
\min \left\{\left(\mu_{1} \times \mu_{2}\right)\left(x_{1}, x_{2}\right)\right. & \left.,\left(\mu_{1} \times \mu_{2}\right)\left(y_{1}, y_{2}\right)\right\} \\
= & \min \left\{\min \left\{\mu_{1}\left(x_{1}\right), \mu_{2}\left(x_{2}\right)\right\}, \min \left\{\mu_{1}\left(y_{1}\right), \mu_{2}\left(y_{2}\right)\right\}\right\} \\
= & \min \left\{\min \left\{\mu_{1}\left(x_{1}\right), \mu_{1}\left(y_{1}\right)\right\}, \min \left\{\mu_{2}\left(x_{2}\right), \mu_{2}\left(y_{2}\right)\right\}\right\} \\
& \geq \min \left\{\mu_{1}\left(x_{1} * y_{1}\right), \mu_{2}\left(x_{2} * y_{2}\right)\right\} \\
= & \left(\mu_{1} \times \mu_{2}\right)\left(x_{1} * y_{1}, x_{2} * y_{2}\right) \\
= & \left(\mu_{1} \times \mu_{2}\right)\left(\left(x_{1}, x_{2}\right) *\left(y_{1}, y_{2}\right)\right)
\end{aligned}
$$

and hence the condition $\left(F_{3}\right)$ holds. Thus $\mu_{1} \times \mu_{2}$ is a fuzzy quick ideal of $X_{1} \times X_{2}$.

For an endomorphism $f$ of a $d$-algebra $X$ and a fuzzy set $\mu$ in $X$, we define a new fuzzy set $\mu^{f}$ by $\mu^{f}(x)=\mu(f(x))$ for all $x \in X$. Note that 
for any endomorphism $f$ of a $d$-algebra $X$, we have $f(0)=0$ for any $x \in X$, since $f(0 * 0)=f(0) * f(0)=0$.

Proposition 4.14. If $\mu$ is a fuzzy quick ideal of a $d$-algebra $X$, then so is $\mu^{f}$.

Proof. For any $x \in X$, we have $\mu^{f}(0)=\mu(f(0)) \geq \mu^{f}(x)=\mu(f(x))$. The condition $\left(F_{0}\right)$ is true. For any $x, y \in X$ with $x * y \neq 0$, we have

$$
\begin{aligned}
\min \left\{\mu^{f}(x), \mu^{f}(y)\right\} & =\min \{\mu(f(x)), \mu(f(y))\} \\
& \geq \mu(f(x) * f(y)) \\
& =\mu(f(x * y)) \\
& =\mu^{f}(x * y) .
\end{aligned}
$$

The condition $\left(F_{3}\right)$ holds. This competes the proof.

Example 4.15. Let $X:=\{0, a, b, c\}$ be a $d$-algebra as in Example 3.4-(1). Then $A=\{0, a\}$ is a quick ideal of $X$. Let $\mu: X \rightarrow[0,1]$ be a fuzzy set defined by $\mu(0)=\mu(a)=t_{1}$ and $\mu(b)=\mu(c)=t_{2}$ for $t_{1}, t_{2} \in[0,1]$ with $t_{1}>t_{2}$. Then $\mu$ is a fuzzy quick ideal of $X$. There exist 5 endomorphisms of a $d$-algebra $X$ as follows:

$$
\begin{aligned}
& f_{1}: 0 \rightarrow 0, a \rightarrow 0, b \rightarrow 0, c \rightarrow 0 \\
& f_{2}: 0 \rightarrow 0, a \rightarrow 0, b \rightarrow 0, c \rightarrow a \\
& f_{3}: 0 \rightarrow 0, a \rightarrow 0, b \rightarrow 0, c \rightarrow b \\
& f_{4}: 0 \rightarrow 0, a \rightarrow 0, b \rightarrow 0, c \rightarrow c \\
& f_{5}: 0 \rightarrow 0, a \rightarrow a, b \rightarrow b, c \rightarrow c .
\end{aligned}
$$

By Proposition 4.14, the maps $\mu^{f_{i}}(i=1,2,3,4,5)$ are fuzzy quick ideals of $X$.

Definition 4.16. A fuzzy quick ideal $I$ of a $d$-algebra of $X$ is said to be characteristic if $f(I)=I$ for all $f \in \operatorname{Aut}(X)$, where $\operatorname{Aut}(X)$ is the set of all automorphisms of $X$.

Definition 4.17. A fuzzy quick ideal of a $d$-algebra $X$ is said to be fuzzy characteristic if $\mu^{f}(x)=\mu(x)$ for all $x \in X$ and $f \in \operatorname{Aut}(x)$.

Example 4.18. In Example 4.15, $f_{5}$ is an automorphism of $X$. It is easy to check that $f_{5}(\mu(x))=\mu(x)$ for all $x \in X$. Hence $\mu$ is characteristic. Also $\mu^{f_{5}}(x)=\mu\left(f_{5}(x)\right)=\mu(x)$ for all $x \in X$. Therefore $\mu$ is fuzzy characteristic.

\section{Intuitionistic fuzzy quick ideals in $d$-algebras}


Definition 5.1. ([5]) Let $X$ be a $d$-algebra. An IFS $D=<x, \mu_{D}, \gamma_{D}>$ in $X$ is called an intuitionistic fuzzy d-algebra if satisfies:

$$
\mu_{D}(x * y) \geq \min \left\{\mu_{D}(x), \mu_{D}(y)\right\} \text { and } \gamma_{D}(x * y) \leq \max \left\{\gamma_{D}(x), \gamma_{D}(y)\right\}
$$

for all $x, y \in X$.

Definition 5.2. Let $X$ be a $d$-algebra. An IFS $D=\left\langle x, \mu_{D}, \gamma_{D}\right\rangle$ in $X$ is called an intuitionistic fuzzy $B C K$-ideal of $X$ if satisfies:

$\left(a_{0}\right)(\forall x \in X)\left(\mu_{D}(0) \geq \mu_{D}(x), \gamma_{D}(0) \leq \gamma_{D}(x)\right)$,

$\left(a_{1}\right)(\forall x, y \in X)\left(\mu_{D}(x) \geq \min \left\{\mu_{D}(x * y), \mu_{D}(y)\right\}\right.$ and $\gamma_{D}(x) \leq \max \left\{\gamma_{D}(x *\right.$ $\left.\left.y), \gamma_{D}(y)\right\}\right)$.

An IFS $D=<x, \mu_{D}, \gamma_{D}>$ in $X$ is called an intuitionistic fuzzy d-ideal of $X$ if satisfies $\left(a_{1}\right)$ and

$\left(a_{2}\right)(\forall x, y \in X)\left(\mu_{D}(x * y) \geq \mu_{D}(x)\right.$ and $\left.\gamma_{D}(x * y) \leq \gamma_{D}(x)\right)$.

An IFS $D=<x, \mu_{D}, \gamma_{D}>$ in $X$ is called an intuitionistic fuzzy quick ideal of $X$ if satisfies $\left(a_{0}\right)$ and

$\left(a_{3}\right)(\forall x, y \in X$ with $x * y \neq 0)\left(\min \left\{\mu_{D}(x), \mu_{D}(y)\right\} \geq \mu_{D}(x * y)\right.$ and $\left.\gamma_{D}(x * y) \geq \max \left\{\gamma_{D}(x), \gamma_{D}(y)\right\}\right)$.

Example 5.3. Let $X:=\{0,1,2,3,4\}$ be a $d$-algebra which is not a $B C K$-algebra with the following Cayley table:

\begin{tabular}{l|lllll}
$*$ & 0 & 1 & 2 & 3 & 4 \\
\hline 0 & 0 & 0 & 0 & 0 & 0 \\
1 & 1 & 0 & 0 & 1 & 0 \\
2 & 2 & 2 & 0 & 0 & 2 \\
3 & 3 & 3 & 3 & 0 & 0 \\
4 & 4 & 3 & 3 & 1 & 0
\end{tabular}

Let $D=<x, \mu_{D}(x), \gamma_{D}(x)>$ be an IFS in $X$ defined by $\mu_{D}(0)=$ $\mu_{D}(1)=0.5, \mu_{D}(2)=\mu_{D}(3)=\mu_{D}(4)=0.03, \gamma_{D}(0)=\gamma_{D}(1)=0.05$, $\gamma_{D}(2)=\gamma_{D}(3)=\gamma_{D}(4)=0.3$. Then $D=<x, \mu_{D}(x), \gamma_{D}(x)>$ is an intuitionistic fuzzy $B C K$-ideal of $X$ and an intuitionistic fuzzy $d$-algebra of $X$. 
Example 5.4. Let $X:=\{0,1,2,3,4\}$ be a $d$-algebra which is not a $B C K$-algebra with the following Cayley table:

\begin{tabular}{l|lllll}
$*$ & 0 & 1 & 2 & 3 & 4 \\
\hline 0 & 0 & 0 & 0 & 0 & 0 \\
1 & 1 & 0 & 0 & 1 & 0 \\
2 & 2 & 2 & 0 & 0 & 2 \\
3 & 3 & 3 & 3 & 0 & 3 \\
4 & 4 & 3 & 3 & 3 & 0
\end{tabular}

Let $D=<x, \mu_{D}(x), \gamma_{D}(x)>$ be an IFS in $X$ defined by $\mu_{D}(3)=$ $0.03, \mu_{D}(x)=0.5, \gamma_{D}(3)=0.3$, and $\gamma_{D}(x)=0.05$ for $x \neq 3$. Then $D=<x, \mu_{D}(x), \gamma_{D}(x)>$ is an intuitionistic fuzzy $B C K$-ideal of $X$ which is not an intuitionistic fuzzy $d$-ideal of $X$ since $\mu_{D}(4 * 3)=\mu_{D}(3)=$ $0.03 \leq \mu_{D}(4)=0.5$ and $\gamma_{D}(4 * 3)=\gamma_{D}(3)=0.3 \geq \gamma_{D}(4)=0.05$.

Example 5.5. Let $X:=\{0, a, b, c\}$ be a $d$-algebra as in Example 3.4(2). Let $D=<x, \mu_{D}(x), \gamma_{D}(x)>$ be an IFS in $X$ defined by $\mu_{D}(b)=$ $0.3, \mu_{D}(x)=0.8, \gamma_{D}(b)=0.08$, and $\gamma_{D}(x)=0.03$ for $x \neq b$. Then $D=<x, \mu_{D}(x), \gamma_{D}(x)>$ is an intuitionistic fuzzy quick ideal of $X$, but not an intuitionistic fuzzy $B C K$-ideal of $X$ since $\mu_{D}(b)=0.3 \leq$ $\min \left\{\mu_{D}(b * c)=\mu_{D}(0), \mu_{D}(c)\right\}=0.8$ and $\gamma_{D}(b)=0.08 \geq \max \left\{\gamma_{D}(b *\right.$ $\left.c)=\gamma_{D}(0), \gamma_{D}(c)\right\}=0.03$. Also $D$ is an intuitionistic fuzzy $d$-algebra.

Example 5.6. Let $X:=\{0, a, b, c\}$ be a $d$-algebra as in Example 3.4(1). Let $D=<x, \mu_{D}(x), \gamma_{D}(x)>$ be an IFS in $X$ defined by $\mu_{D}(c)=$ $0.2, \mu_{D}(x)=0.7, \gamma_{D}(c)=0.08$, and $\gamma_{D}(x)=0.01$ for $x \neq c$. Then $D=<x, \mu_{D}(x), \gamma_{D}(x)>$ is an intuitionistic fuzzy $B C K$-ideal of $X$, but not an intuitionistic fuzzy quick ideal of $X$ since $a * c=b \neq 0$, $\min \left\{\mu_{D}(a), \mu_{D}(c)\right\}=0.2<\mu_{D}(a * c)=\mu_{D}(b)=0.7$ and $\gamma_{D}(a * c)=$ $\gamma_{D}(b)=0.01<\max \left\{\gamma_{D}(a), \gamma_{D}(c)\right\}=0.08$.

Example 5.7. Let $X:=\{0, a, b, c\}$ be a $d$-algebra as in Examle 3.4-(3). Let $D=<x, \mu_{D}(x), \gamma_{D}(x)>$ be an IFS in $X$ defined by $\mu_{D}(b)=0.3, \mu_{D}(x)=0.8, \gamma_{D}(b)=0.08$, and $\gamma_{D}(x)=0.03$ for $x \neq b$. Then $D=<x, \mu_{D}(x), \gamma_{D}(x)>$ is an intuitionistic fuzzy quick ideal of $X$, but not an intuitionistic fuzzy $d$-algebra of $X$ since $\mu_{D}(a * c)=$ $\mu_{D}(b)=0.3<\min \left\{\mu_{D}(a), \mu_{D}(c)\right\}=0.8$ and $\gamma_{D}(a * c)=\gamma_{D}(b)=0.08>$ $\max \left\{\gamma_{D}(a), \gamma_{D}(c)\right\}=0.03$. Also $D=<x, \mu_{D}(x), \gamma_{D}(x)>$ is not an intuitionistic fuzzy $B C K$-ideal of $X$, since $\mu_{D}(b)=0.3<\min \left\{\mu_{D}(b * c)=\right.$ $\left.\mu_{D}(0)=0.8, \mu_{D}(c)=0.8\right\}=0.8$ and $\gamma_{D}(b)=0.08>\max \left\{\gamma_{D}(b * c)=\right.$ $\left.\gamma_{D}(0)=0.03, \gamma_{D}(c)=0.03\right\}=0.03$. 
Theorem 5.8. Let $D=<x, \mu_{D}(x), \gamma_{D}(x)>$ be an intuitionistic fuzzy $B C K$-ideal of a d-algebra $X$. Then

(i) $\mu_{D}$ is order reversing,

(ii) $x * y \leq z$ implies $\gamma_{D}(x * z) \leq \gamma_{D}(z)$ for all $x, y, z \in X$.

Proof. (i) Let $x, y \in X$ with $x \leq y$. Then $x * y=0$. Hence

$$
\mu_{D}(x) \geq \min \left\{\mu_{D}(x * y)=\mu_{D}(0), \mu_{D}(y)\right\}=\mu_{D}(y) .
$$

Thus $\mu_{D}(x) \geq \mu_{D}(y)$.

(ii) For all $x, y, z \in X$, we have

$$
\begin{aligned}
\gamma_{D}(x * y) & \leq \max \left\{\gamma_{D}((x * y) * z), \gamma_{D}(z)\right\} \\
& =\max \left\{\gamma_{D}(0), \gamma_{D}(z)\right\}=\gamma_{D}(z)
\end{aligned}
$$

Therefore $\gamma_{D}(x * y) \leq \gamma_{D}(z)$.

Theorem 5.9. Let $D=<x, \mu_{D}(x), \gamma_{D}(x)>$ be an intuitionistic fuzzy $B C K$-ideal of a $d$-algebra $X$. Then $x * y \leq z$ implies $\mu_{D}(x) \geq$ $\min \left\{\mu_{D}(y), \mu_{D}(z)\right\}$ and $\gamma_{D}(x) \leq \max \left\{\gamma_{D}(y), \gamma_{D}(z)\right\}$ for all $x, y, z \in X$.

Proof. Let $x, y, z \in X$ such that $x * y \leq z$. Then $(x * y) * z=0$. Hence

$$
\begin{aligned}
\mu_{D}(x * y) & \geq \min \left\{\mu_{D}((x * y) * z), \mu_{D}(z)\right\} \\
& =\min \left\{\mu_{D}(0), \mu_{D}(z)\right\}=\mu_{D}(z) .
\end{aligned}
$$

Therefore we have

$$
\begin{aligned}
\mu_{D}(x) & \geq \min \left\{\mu_{D}(x * y), \mu_{D}(y)\right\} \\
& \geq \min \left\{\mu_{D}(z), \mu_{D}(y)\right\} .
\end{aligned}
$$

Thus $\mu_{D}(x) \geq \min \left\{\mu_{D}(z), \mu_{D}(y)\right\}$.

By a similar way, we obtain $\gamma_{D}(x) \leq \max \left\{\gamma_{D}(y), \gamma_{D}(z)\right\}$ for all $x, y, z \in X$.

Theorem 5.10. If $\left\{D_{i} \mid i \in \Lambda\right\}$ is an arbitrary family of intuitionistic fuzzy quick ideal of a $d$-algebra $X$, then $\cap D_{i}$ is an intuitionistic fuzzy quick ideal of $X$, where $\cap D_{i}=\left\{\left\langle x, \wedge \mu_{D_{i}}(x), \vee \gamma_{D_{i}}(x)\right\rangle \mid x \in X\right\}$.

Proof. Since $\mu_{D_{i}}(0) \geq \mu_{D_{i}}(x)$ and $\gamma_{D_{i}}(0) \leq \gamma_{D_{i}}(x)$ for all $x \in X$, we have $\wedge \mu_{D_{i}}(0) \geq \wedge \mu_{D_{i}}(x)$ and $\vee \gamma_{D_{i}}(0) \leq \vee \gamma_{D_{i}}(x)$ for all $x \in X$.

Let $x, y \in X$ with $x * y \neq 0$. Then

$$
\begin{aligned}
\wedge \mu_{D_{i}}(x * y) & \leq \wedge\left(\min \left\{\mu_{D_{i}}(x), \mu_{D_{i}}(y)\right\}\right) \\
& =\min \left\{\wedge \mu_{D_{i}}(x), \wedge \mu_{D_{i}}(y)\right\}
\end{aligned}
$$

and

$$
\begin{aligned}
\vee \gamma_{D_{i}}(x * y) & \geq \vee\left(\max \left\{\gamma_{D_{i}}(x), \gamma_{D_{i}}(y)\right\}\right) \\
& =\max \left\{\vee \gamma_{D_{i}}(x), \vee \gamma_{D_{i}}(y)\right\}
\end{aligned}
$$


Hence $\cap D_{i}=\left\{\left\langle x, \wedge \mu_{D_{i}}(x), \vee \gamma_{D_{i}}(x)>\right| x \in X\right\}$ is an intuitionistic fuzzy quick ideal of $X$.

Theorem 5.11. If $D=<x, \mu_{D}, \gamma_{D}>$ is an intuitonistic fuzzy quick ideal of $X$, then so is $\square D$, where $\square D=\left\{<x, \mu_{D}(x), 1-\mu_{D}(x)>\mid x \in\right.$ $X\}$.

Proof. It is sufficient to show that $\overline{\mu_{D}}(x):=1-\mu_{D}(x)$ satisfies the second condition of $\left(a_{3}\right)$ in Definition 5.2. Let $x, y \in X$ with $x * y \neq 0$. Then

$$
\begin{aligned}
\overline{\mu_{D}}(x * y) & =1-\mu_{D}(x * y) \\
& \geq 1-\min \left\{\mu_{D}(x), \mu_{D}(y)\right\} \\
& =\max \left\{1-\mu_{D}(x), 1-\mu_{D}(y)\right\} \\
& =\max \left\{\overline{\mu_{D}}(x), \overline{\mu_{D}}(y)\right\} .
\end{aligned}
$$

Hence $\square D$ is an intuitionistic fuzzy quick ideal of $X$.

Theorem 5.12. If an IFS $D=<x, \mu_{D}, \gamma_{D}>$ in $X$ is an intuitionistic fuzzy quick ideal of $X$, then the sets

$$
X_{\mu}:=\left\{x \in X \mid \mu_{D}(x)=\mu_{D}(0)\right\} \text { and } X_{\gamma}:=\left\{x \in X \mid \gamma_{D}(x)=\gamma_{D}(0)\right\}
$$

are quick ideals of $X$.

Proof. Clearly, $0 \in X_{\mu}$. Let $x, y \in X$ with $x * y \neq 0, x * y \in X_{\mu}$. Then $\mu_{D}(x * y)=\mu_{D}(0)$. Hence $\min \left\{\mu_{D}(x), \mu_{D}(y)\right\} \geq \mu_{D}(x * y)=\mu_{D}(0)$. By $\left(a_{0}\right)$, we have $\mu_{D}(x)=\mu_{D}(y)=\mu_{D}(0)$. Thus $x, y \in X_{\mu}$.

By the definition of $X_{\gamma}, 0 \in X_{\gamma}$. Let $x, y \in X$ with $x * y \neq$ $0, x * y \in X_{\gamma}$. Then $\gamma_{D}(x * y)=\gamma_{D}(0)$. Hence $\gamma_{D}(0)=\gamma_{D}(x * y) \geq$ $\max \left\{\gamma_{D}(x), \gamma_{D}(y)\right\}$. By $\left(a_{0}\right)$, we have $\gamma_{D}(x)=\gamma_{D}(y)=\gamma_{D}(0)$. Thus $x, y \in X_{\gamma}$.

Definition 5.13. Let $D=<x, \mu_{D}, \gamma_{D}>$ be an IFS in $X$ and let $t \in[0,1]$. Then the set

$U\left(\mu_{D} ; t\right):=\left\{x \in X \mid \mu_{D}(x) \geq t\right\}$ (resp., $\left.L\left(\gamma_{D} ; t\right):=\left\{x \in X \mid \gamma_{D}(x) \leq t\right\}\right)$ is called a $\mu$-level t-cut (resp., $\gamma$-level $t$-cut) of $D$.

Theorem 5.14. If an IFS $D=<x, \mu_{D}, \gamma_{D}>$ in $X$ is an intuitionistic fuzzy quick ideal of $X$, then the $\mu$-level $t$-cut and $\gamma$-revel-cut of $D$ are quick ideals of $X$ for every $t \in[0,1]$ such that $t \in \operatorname{Im}\left(\mu_{D}\right) \cap \operatorname{Im}\left(\gamma_{D}\right)$, which are called a $\mu$-level quick ideal and a $\gamma$-level quick ideal of $X$, respectively.

Proof. By $\left(a_{0}\right)$, we have $0 \in U\left(\mu_{D} ; t\right)$ and $0 \in L\left(\gamma_{D} ; t\right)$. 
Let $x, y \in X$ with $x * y \neq 0, x * y \in U\left(\mu_{D} ; t\right)$. Then $\mu_{D}(x * y) \geq t$ and so $\min \left\{\mu_{D}(x), \mu_{D}(y)\right\} \geq \mu_{D}(x * y) \geq t$. Hence $x, y \in U\left(\mu_{D} ; t\right)$.

Let $x, y \in X$ with $x * y \neq 0, x * y \in L\left(\gamma_{D} ; t\right)$. Then $\gamma_{D}(x * y) \leq t$ and so $t \geq \gamma_{D}(x * y) \geq \max \left\{\gamma_{D}(x), \gamma_{D}(y)\right\}$. Hence $x, y \in L\left(\mu_{D} ; t\right)$.

Theorem 5.15. Let $D=<x, \mu_{D}, \gamma_{D}>$ be an IFS in $X$ such that the sets $U\left(\mu_{D} ; t\right)$ and $L\left(\gamma_{D} ; t\right)$ are quick ideals of $X$. Then $D=<x, \mu_{D}, \gamma_{D}>$ is an intuitionistic fuzzy quick ideal of $X$

Proof. Assume that there exists $x_{0}, y_{0} \in X$ with $x_{0} * y_{0} \neq 0$ such that $\min \left\{\mu_{D}\left(x_{0}\right), \mu_{D}\left(y_{0}\right)\right\}<\mu_{D}\left(x_{0} * y_{0}\right)$. Let $t_{0}:=\frac{1}{2}\left(\mu_{D}\left(x_{0} * y_{0}\right)+\right.$ $\left.\min \left\{\mu_{D}\left(x_{0}\right), \mu_{D}\left(y_{0}\right)\right\}\right)$. Then $\min \left\{\mu_{D}\left(x_{0}\right), \mu_{D}\left(y_{0}\right)\right\}<t_{0}<\mu_{D}\left(x_{0} * y_{0}\right)$ and so $x_{0}, y_{0} \notin U\left(\mu_{D} ; t_{0}\right)$ and $x_{0} * y_{0} \in U\left(\mu_{D} ; t_{0}\right)$. This is a contradiction, and therefore $\min \left\{\mu_{D}(x), \mu_{D}(y)\right\} \geq \mu_{D}(x * y)$ for all $x, y \in X$. Now suppose that there exists $x_{0}, y_{0} \in X$ with $x_{0} * y_{0} \neq 0$ such that $\gamma_{D}\left(x_{0} * y_{0}\right)<\max \left\{\gamma_{D}\left(x_{0}\right), \gamma_{D}\left(y_{0}\right)\right\}$. Taking $s_{0}:=\frac{1}{2}\left(\gamma_{D}\left(x_{0} * y_{0}\right)+\right.$ $\left.\max \left\{\gamma_{D}\left(x_{0}\right), \gamma_{D}\left(y_{0}\right)\right\}\right)$, then $\gamma_{D}\left(x_{0} * y_{0}\right)<s_{0}<\max \left\{\gamma_{D}\left(x_{0}\right), \gamma_{D}\left(y_{0}\right)\right\}$. It follows that $x_{0} * y_{0} \in L\left(\gamma_{D} ; s_{0}\right)$ and $x_{0}, y_{0} \notin L\left(\gamma_{D} ; s_{0}\right)$. This is a contradiction. Hence

$$
\gamma_{D}(x * y) \geq \max \left\{\gamma_{D}(x), \gamma_{D}(y)\right\}
$$

for all $x, y \in X$. This completes the proof.

Theorem 5.16. Any quick ideal of a $d$-algebra $X$ can be realized as both a $\mu$-level quick ideal and $\gamma$-level quick ideal of some intuitionistic fuzzy quick ideal of $X$.

Proof. Let $S$ be a quick ideal of $X$ and let $\mu_{D}$ and $\gamma_{D}$ be fuzzy sets in $X$ defined by

and

$$
\mu_{D}(x):= \begin{cases}t_{1}, & \text { if } x \in S \\ t_{2}, & \text { otherwise }\end{cases}
$$

$$
\gamma_{D}(x):= \begin{cases}s_{1}, & \text { if } x \in S \\ s_{2}, & \text { otherwise }\end{cases}
$$

for all $x \in X$ where $t_{i}$ and $s_{i}$ are fixed numbers in $(0,1)$ such that $t_{1}>$ $t_{2}, s_{1}<s_{2}$, and $t_{i}+s_{i}<1$ for $i=1,2$. Since $0 \in S$, we have $\mu_{D}(0)=t_{1}$ and $\gamma_{D}(0)=s_{1}$. Hence $\mu_{D}(0) \geq \mu_{D}(x)$ and $\gamma_{D}(0) \leq \gamma_{D}(x), \forall x \in X$.

Let $x, y \in X$ with $x * y \neq 0$. If $x * y \in S$, then $x, y \in S$. Hence $\mu_{D}(x * y)=\min \left\{\mu_{D}(x), \mu_{D}(y)\right\}$ and $\gamma_{D}(x * y)=\max \left\{\gamma_{D}(x), \gamma_{D}(y)\right\}$. If $x * y \notin S$, then $\mu_{D}(x * y)=t_{2}$ and $\gamma_{D}(x * y)=s_{2}$. Hence $t_{2}=\mu_{D}(x * y) \leq$ $\min \left\{\mu_{D}(x), \mu_{D}(y)\right\}$ and $s_{2}=\gamma_{D}(x * y) \geq \max \left\{\gamma_{D}(x), \gamma_{D}(y)\right\}$. Therefore 
$D=<x, \mu_{D}, \gamma_{D}>$ is an intuitionistic fuzzy quick ideal of $X$. Obviously, $U\left(\mu_{D} ; t\right)=S=L\left(\gamma_{D} ; s\right)$. This completes the proof.

Theorem 5.17. Let $\alpha$ be a $d$-homomorphism of a $d$-algebra $X$ into a $d$-algebra $Y$ and $B$ an intuitionistic quick ideal of $Y$, Then $\alpha^{-1}(B)$ is an intuitionistic fuzzy quick ideal of $X$.

Proof. For all $x \in X$, we have

$$
\begin{aligned}
\mu_{\alpha^{-1}(B)}(0) & =\mu_{B}(\alpha(0)) \geq \mu_{B}(\alpha(x)) \\
& =\mu_{\alpha^{-1}(B)}(x)
\end{aligned}
$$

and

$$
\begin{aligned}
\gamma_{\alpha^{-1}(B)}(0) & =\gamma_{B}(\alpha(0)) \leq \gamma_{B}(\alpha(x)) \\
& =\gamma_{\alpha^{-1}(B)}(x) .
\end{aligned}
$$

Let $x, y \in X$ with $x * y \neq 0$. Then

$$
\begin{aligned}
\mu_{\alpha^{-1}(B)}(x * y) & =\mu_{B}(\alpha(x * y))=\mu_{B}(\alpha(x) * \alpha(y)) \\
& \leq \min \left\{\mu_{B}(\alpha(x)), \mu_{B}(\alpha(y))\right\} \\
& =\min \left\{\mu_{\alpha^{-1}(B)}(x), \mu_{\alpha^{-1}(B)}(y)\right\}
\end{aligned}
$$

and

$$
\begin{aligned}
\gamma_{\alpha^{-1}(B)}(x * y) & =\gamma_{B}(\alpha(x * y))=\gamma_{B}(\alpha(x) * \alpha(y)) \\
& \geq \max \left\{\gamma_{B}(\alpha(x)), \gamma_{B}(\alpha(y))\right\} \\
& =\max \left\{\gamma_{\alpha^{-1}(B)}(x), \gamma_{\alpha^{-1}(B)}(y)\right\} .
\end{aligned}
$$

Hence $\alpha^{-1}(B)$ is an intuitionistic fuzzy quick ideal of $X$.

Theorem 5.18. Let $X$ be a d-algebra, $\Lambda$ an non-empty set of $[0,1]$, and $\left\{G_{i} \mid i \in \Lambda\right\}$ be a collection of quick ideals of $X$ such that

(i) $X=\cup_{i \in \Lambda} G_{i}$,

(ii) $s<t$ if and only if $G_{t} \subseteq G_{s}$ for all $s, t \in \Lambda$.

Then an IFS $D=<x, \mu_{D}, \gamma_{D}>$ of $X$ defined by $\mu_{D}(x):=\sup \{s \in$ $\left.\Lambda \mid x \in G_{s}\right\}$ and $\gamma_{D}(x):=\inf \left\{s \in \Lambda \mid x \in G_{s}\right\}$ is an intuitionistic fuzzy quick ideal of $X$.

Proof. Using Theorem 5.16, it is sufficient to show that $U\left(\mu_{D} ; s\right)$ and $L\left(\gamma_{D} ; t\right)$ are quick ideals of $X$ for all $s \in\left[0, \mu_{D}(0)\right]$ and $t \in\left[\gamma_{D}(0), 1\right]$. In order to prove that $U\left(\mu_{D} ; s\right)$ is a quick ideal of $X$, we divide into the following cases:

(i) $s=\sup \{q \in \Lambda \mid q<s\}$, and (ii) $s \neq \sup \{q \in \Lambda \mid q<s\}$.

Case (i) implies that $x \in U\left(\mu_{D} ; s\right) \Leftrightarrow x \in G_{q}$ for all $q<s \Leftrightarrow x \in$ $\cap_{q<s} G_{q}$, so that $U\left(\mu_{D} ; s\right)=\cap_{q<s} G_{q}$, which is a quick ideal of $X$. 
For the case (ii), we claim that $U\left(\mu_{D} ; s\right)=\cup_{q \geq s} G_{q}$. If $x \in \cup_{q \geq s} G_{q}$, then $x \in G_{q}$ for some $q \geq s$. Hence $\mu_{D}(x) \geq q \geq s$, so that $x \in U\left(\mu_{D} ; s\right)$. This proves that $\cap_{q \geq s} G_{q} \subseteq U\left(\mu_{D} ; s\right)$.

Now assume that $x \notin U_{q \geq s} G_{q}$. Then $x \notin G_{q}$ for all $q \geq s$. Since $s \neq \sup \{q \in \Lambda \mid q<s\}$, there exists $\epsilon>0$ such that $(s-\epsilon, s) \cap \Lambda=\emptyset$. Hence $x \notin G_{q}$ for all $q>s-\epsilon$, which means that if $x \in G_{q}$, then $q \leq s-\epsilon$. Therefore $U\left(\mu_{D} ; s\right) \subseteq U_{q \geq s} G_{q}$, and thus $U\left(\mu_{D} ; s\right)=\cup_{q \geq s} G_{q}$, which is a quick ideal of $X$.

Next, we prove that $L\left(\gamma_{D} ; t\right)$ is a quick ideal of $X$ for all $t \in\left[\gamma_{D}(0), 1\right]$. We consider the following two cases:

$$
\text { (iii) } \mathrm{t}=\inf \{\mathrm{q} \in \Lambda \mid \mathrm{t}<\mathrm{q}\} \text {, and (iv) } \mathrm{t} \neq \inf \{\mathrm{q} \in \Lambda \mid \mathrm{t}<\mathrm{q}\} \text {. }
$$

For the case (iii) we have $x \in L\left(\gamma_{D} ; t\right) \Leftrightarrow x \in G_{q}$ for all $t<q \Leftrightarrow x \in$ $\cap_{t<q} G_{q}$ and hence $L\left(\gamma_{D} ; t\right)=\cap_{t<q} G_{q}$, which is a quick ideal of $X$.

For the case (iv), there exists $\epsilon>0$ such that $(t, t+\epsilon) \cap \Lambda=\emptyset$. We will show that $L\left(\gamma_{D} ; t\right)=\cup_{t \geq q} G_{q}$.

If $x \in \cup_{t \geq q} G_{q}$, then $x \in G_{q}$ for some $t \geq q$. It follows that $\gamma_{D}(x) \leq$ $q \leq t$ so that $x \in L\left(\gamma_{D} ; t\right)$. Hence $\cup_{t \geq q} G_{q} \subseteq L\left(\gamma_{D} ; t\right)$.

Conversely, if $x \notin \cup_{t \geq q} G_{q}$, then $x \notin G_{q}$ for all $q \geq t$, which implies that $x \notin G_{q}$ for all $q<t+\epsilon$, that is, if $x \in G_{q}$, then $q \geq t+\epsilon$. Thus $\gamma_{D}(x) \geq t+\epsilon>t$, i.e., $x \notin L\left(\gamma_{D} ; t\right)$. Therefore $L\left(\gamma_{D} ; t\right) \subseteq \cup_{t \geq q} G_{q}$ and consequently $L\left(\gamma_{D} ; t\right)=\cup_{t \geq q} G_{q}$ which is a quick ideal of $X$. This competes the proof.

Let $\operatorname{IF}(X)$ be the family of all intuitionistic fuzzy quick ideals of a $d$-algebra $X$ and let $t \in[0,1]$. Define binary relations $U^{t}$ and $L^{t}$ on $I F(X)$ as follows:

$$
(A, B) \in U^{t} \quad \Longleftrightarrow \quad U\left(\mu_{A} ; t\right)=U\left(\mu_{B} ; t\right)
$$

and

$$
(A, B) \in L^{t} \quad \Longleftrightarrow \quad L\left(\gamma_{A} ; t\right)=L\left(\gamma_{B} ; t\right),
$$

respectively, for $A=<x, \mu_{A}, \gamma_{A}>$ and $\left.B=<x, \mu_{B}, \gamma_{B}\right)$ in $\operatorname{IF}(X)$. Then clearly $U^{t}$ and $L^{t}$ are equivalence relations on $\operatorname{IF}(X)$.

For any $A=<x, \mu_{A}, \gamma_{A}>\in I F(X)$, let $[A]_{U^{t}}$ (resp., $[A]_{L^{t}}$ ) denote the equivalence class of a modulo $U^{t}$ (resp., $L^{t}$ ), and denoted by $I F(X) / U^{t}$ (resp., $I F(X) / L^{t}$ ) the collection of all equivalence classes modulo $U^{t}$ (resp., $\left.L^{t}\right)$; so

$$
\begin{gathered}
\operatorname{IF}(X) / U^{t}:=\left\{[A]_{U^{t}} \mid A=<x, \mu_{A}, \gamma_{A}>\in I F(X)\right\} \\
\text { (resp., } \left.\quad I F(X) / L^{t}:=\left\{[A]_{L^{t}} \mid A=<x, \mu_{A}, \gamma_{A}>\in I F(X)\right\}\right) .
\end{gathered}
$$


Now let $I(X)$ denote the family of all quick ideals of $X$ and let $t \in[0,1]$. Define maps $f_{t}$ and $g_{t}$ from $I F(X)$ to $I(X) \cup\{\emptyset\}$ by $f_{t}(A)=U\left(\mu_{A} ; t\right)$ and $g_{t}(A)=L\left(\gamma_{A} ; t\right)$, respectively, for all $A=<x, \mu_{A}, \gamma_{A}>\in I F(X)$. Then $f_{t}$ and $g_{t}$ are clearly well-defined.

Theorem 5.19. For any $t \in(0,1)$ the maps $f_{t}$ and $g_{t}$ are surjective from $I F(X)$ to $I(X) \cup\{\emptyset\}$.

Proof. Let $t \in(0,1)$. Note that $\mathbf{0}_{\sim}=\langle x, \mathbf{0}, \mathbf{1}\rangle$ is in $\operatorname{IF}(X)$, where $\mathbf{0}$ and $\mathbf{1}$ are fuzzy sets in $X$ defined by $\mathbf{0}(x)=0$ and $\mathbf{1}(x)=x$ for all $x \in X$. Obviously $f_{t}\left(\mathbf{0}_{\sim}\right)=U(\mathbf{0} ; t)=\emptyset=L(\mathbf{1} ; t)=g_{t}\left(\mathbf{0}_{\sim}\right)$. Let $\emptyset \neq G \in I(X)$. For $G_{\sim}=\left\langle x, \chi_{G}, \overline{\chi_{G}}>\in I F(X)\right.$, we have $f_{t}\left(G_{\sim}\right)=U\left(\chi_{G} ; t\right)$ and $g_{t}\left(G_{\sim}\right)=L\left(\overline{\chi_{G}} ; t\right)=G$. Hence $f_{t}$ and $g_{t}$ are surjective.

Theorem 5.20. The quotient sets $I F(X) / U^{t}$ and $I F(X) / L^{t}$ are equipotent to $I(X) \cup\{\emptyset\}$ for every $t \in(0,1)$.

Proof. For $t \in(0,1)$ let $f_{t}^{*}$ (resp., $g_{t}^{*}$ ) be a map from $I F(X) / U^{t}$ (resp., $I F(X) / L^{t}$ ) to $I(X) \cup\{\emptyset\}$ defined by $f_{t}^{*}\left([A]_{U^{t}}\right)=f_{t}(A)$ (resp., $\left.g_{t}^{*}\left([A]_{L^{t}}\right)=g_{t}(A)\right)$ for all $A=<x, \mu_{A}, \mu_{A}>\in \operatorname{IF}(X)$. If $U\left(\mu_{A} ; t\right)=$ $U\left(\mu_{B} ; t\right)$ and $L\left(\gamma_{A} ; t\right)=L\left(\gamma_{B} ; t\right)$ for $A=<x, \mu_{A}, \gamma_{A}>$ and $B=<$ $x, \mu_{B}, \gamma_{B}>$ in $I F(X)$, then $(A, B) \in U^{t}$ and $(A, B) \in L^{t}$; hence $[A]_{U^{t}}=$ $[B]_{U^{t}}$ and $[A]_{L^{t}}=[B]_{L^{t}}$. Therefore the maps $f_{t}^{*}$ and $g_{t}^{*}$ are injective. Now let $\emptyset \neq G \in I(X)$. For $G_{\sim}=<x, \chi_{G}, \overline{\chi_{G}}>\in I F(X)$, we have

$$
f_{t}^{*}\left(\left[G_{\sim}\right]_{U^{t}}\right)=f_{t}\left(G_{\sim}\right)=U\left(\chi_{G} ; t\right)=G
$$

and

$$
g_{t}^{*}\left(\left[G_{\sim}\right]_{L^{t}}\right)=g_{t}\left(G_{\sim}\right)=L\left(\overline{\chi_{G}} ; t\right)=G .
$$

Finally, for $\mathbf{0}_{\sim}=<x, \mathbf{0}, \mathbf{1}>\in \operatorname{IF}(X)$ we get

$$
f_{t}^{*}\left(\left[\mathbf{0}_{\sim}\right]_{U^{t}}\right)=f_{t}\left(\mathbf{0}_{\sim}\right)=U(\mathbf{0} ; t)=\emptyset
$$

and

$$
g_{t}^{*}\left(\left[\mathbf{0}_{\sim}\right]_{L^{t}}\right)=f_{t}\left(\mathbf{0}_{\sim}\right)=L(\mathbf{1} ; t)=\emptyset .
$$

This shows that $f_{t}^{*}$ and $g_{t}^{*}$ are surjective, and we are done.

For any $t \in[0,1]$, we define another relation $R^{t}$ on $\operatorname{IF}(X)$ as follows:

$$
(A, B) \in R^{t} \Longleftrightarrow U\left(\mu_{A} ; t\right) \cap L\left(\gamma_{A} ; t\right)=U\left(\mu_{B} ; t\right) \cap L\left(\gamma_{B} ; t\right)
$$

for any $A=<x, \mu_{A}, \gamma_{A}>, B=<x, \mu_{B}, \gamma_{B}>\in I F(X)$. Then the relation $R^{t}$ is also an equivalence relation on $\operatorname{IF}(X)$.

Theorem 5.21. For any $t \in(0,1)$, the $\operatorname{map} \phi_{t}: I F(X) \rightarrow I(X) \cup\{\emptyset\}$ defined by $\phi_{t}(A)=f_{t}(A) \cap g_{t}(A)$ for each $A=<x, \mu_{A}, \gamma_{A}>\in I F(X)$ is surjective. 
Proof. Let $t \in(0,1)$. For $\mathbf{0}_{\sim}=<x, \mathbf{0}, \mathbf{1}>\in I F(X)$, we get

$$
\phi_{t}\left(\mathbf{0}_{\sim}\right)=f_{t}\left(\mathbf{0}_{\sim}\right) \cap g_{t}\left(\mathbf{0}_{\sim}\right)=U(\mathbf{0} ; t) \cap L(\mathbf{1} ; t)=\emptyset .
$$

For any $H \in I(X)$, there exists $H_{\sim}=<x, \chi_{H}, \overline{\chi_{H}}>\in I F(X)$ such that

$$
\phi_{t}\left(H_{\sim}\right)=f_{t}\left(H_{\sim}\right) \cap g_{t}\left(H_{\sim}\right)=U\left(\chi_{H} ; t\right) \cap L\left(\overline{\chi_{H}} ; t\right)=H .
$$

This completes the proof.

Theorem 5.22. For any $t \in(0,1)$, the quotient set $I F(X) / R^{t}$ is equipotent to $I(X) \cup\{\emptyset\}$.

Proof. Let $t \in(0,1)$ and let $\phi^{*}: I F(X) / R^{t} \rightarrow I(X) \cup\{\emptyset\}$ be defined by $\phi_{t}^{*}\left([A]_{R^{t}}\right)=\phi_{t}(A)$ for all $[A]_{R^{t}} \in I F(X) / R^{t}$. If $\phi_{t}^{*}\left([A]_{R^{t}}\right)=\phi_{t}^{*}\left([B]_{R^{t}}\right)$ for any $[A]_{R^{t}},[B]_{R^{t}} \in I F(X) / R^{t}$, then $f_{t}(A) \cap g_{t}(A)=f_{t}(B) \cap g_{t}(B)$, i.e., $U\left(\mu_{A} ; t\right) \cap L\left(\gamma_{A} ; t\right)=U\left(\mu_{B} ; t\right) \cap L\left(\gamma_{B} ; t\right)$, hence $(A, B) \in R^{t}$. It follows that $[A]_{R^{t}}=[B]_{R^{t}}$ so that $\phi_{t}^{*}$ is injective. For $\mathbf{0}_{\sim}=<x, \mathbf{0}, \mathbf{1}>\in \operatorname{IF}(X)$,

$$
\phi_{t}^{*}\left(\left[\mathbf{0}_{\sim}\right]_{R^{t}}\right)=\phi_{t}\left(\mathbf{0}_{\sim}\right)=f_{t}\left(\mathbf{0}_{\sim}\right) \cap g_{t}\left(\mathbf{0}_{\sim}\right)=U(\mathbf{0} ; t) \cap L(\mathbf{1} ; t)=\emptyset .
$$

If $H \in I F(X)$, then for $H_{\sim}=<x, \chi_{H}, \overline{\chi_{H}}>\in I F(X)$, we have

$$
\phi_{t}^{*}\left(\left[H_{\sim}\right]_{R^{t}}\right)=\phi_{t}\left(H_{\sim}\right)=f_{t}\left(H_{\sim}\right) \cap g_{t}\left(H_{\sim}\right)=U\left(\chi_{H} ; t\right) \cap L\left(\overline{\chi_{H}} ; t\right)=H .
$$

Hence $\phi_{t}^{*}$ is surjective, completing the proof.

\section{References}

[1] S. S. Ahn, Y. B. Jun and H. S. Kim, Fuzzy quick ideals in $B C K / B C I$-algebras, J. Fuzzy Math. 11 (2003), 631-637.

[2] K. T. Atanassov, Intuitionistic fuzzy sets, Fuzzy sets and Systems 20 (1986), 87-96.

[3] K. Iséki, On BCI-algebras, Mathematics Seminar Notes 8 (1980), 125-130.

[4] K. Iséki and S. Tanaka, An introduction to the theory of $B C K$-algebras, Math. Japonica 23(1) (1978), 1-26.

[5] Y. B. Jun, H. S. Kim and D. S. Yoo, Intuitionistic fuzzy d-algebras, Sci. Math. Japon. 66 (2007), 117-125.

[6] Y. B. Jun, J. Neggers and H. S. Kim, Fuzzy $d$-ideals of $d$-algebras, J. Fuzzy Math. 8(1) (2000), 123-130.

[7] Y. C. Lee and H. S. Kim, On $d^{*}$-subalgebras of $d$-transitive $d^{*}$-algebras, Math. Slovaca 49(1) (1999), 27-33.

[8] J. Meng and Y. B. Jun, BCK-algebras, Kyung Moon Sa, Seoul, 1994.

[9] J. Neggers and H. S. Kim, On d-algebras, Math. Slovaca 49, (1999), 19-26.

[10] J. Neggers, Y. B. Jun and H. S. Kim, On $d$-ideals in $d$-algebras, Math. Slovaca 49(3) (1999), 243-251. 
Sun Shin Ahn

Department of Mathematics Education

Dongguk University, Seoul 100-715 Korea

e-mail:sunshine@dongguk.edu

Gyeong Ho Han

Department of Mathematics Education

Dongguk University, Seoul 100-715, Korea

email: pranchisco1@naver.com 